\title{
Are the beneficial cardiovascular effects of simvastatin and metformin also associated with a hormone-dependent mechanism improving insulin sensitivity?
}

C. Bulcão ${ }^{1}$, F.M.A. Giuffrida ${ }^{1}$, F.F. Ribeiro-Filho ${ }^{1}$ and S.R.G. Ferreira ${ }^{2}$

\author{
${ }^{1}$ Divisão de Endocrinologia, Departamento de Medicina Interna, \\ Escola Paulista de Medicina, Universidade Federal de São Paulo, São Paulo, SP, Brasil \\ ${ }^{2}$ Departamento de Nutrição, Faculdade de Saúde Pública, Universidade de São Paulo, \\ São Paulo, SP, Brasil
}

\section{Correspondence}

C. Bulcão

Disciplina de Endocrinologia

EPM, UNIFESP

Rua Botucatu, 740

04034-970 São Paulo, SP

Brasil

Fax: +55-11-5572-0889

E-mail: cbulcao@uol.com.br

Research supported by FAPESP

(No. 02/10010-9).

.....................

Received April 24, 2006 Accepted October 9, 2006

\begin{abstract}
In addition to lipid-lowering and cardiovascular protective actions, statins may have beneficial effects on insulin sensitivity. The objective of the present study was to evaluate the effect of simvastatin therapy on insulin resistance and on leptin, adiponectin, and Creactive protein (CRP) levels, as compared to metformin, in overweight pre-diabetic subjects. Forty-one subjects with BMI $>25 \mathrm{~kg} / \mathrm{m}^{2}$ and impaired fasting glucose or impaired glucose tolerance were randomized to take simvastatin, $20 \mathrm{mg} / \mathrm{day}(\mathrm{N}=20)$ or metformin, 1.7 $\mathrm{g} /$ day $(\mathrm{N}=21)$ for 16 weeks. Blood samples for the determination of metabolic, hormonal, and inflammatory parameters were obtained at baseline and after each treatment. After metformin therapy, significant reductions in mean BMI and waist circumference were observed, and after simvastatin treatment LDL and triglyceride levels were significantly reduced. Insulin resistance determined by the homeostasis model assessment decreased only with metformin. Independently of the type of medication, a significant decrease in CRP levels was detected from baseline to the end of the study. CRP showed a mean reduction of $0.12 \pm 0.04 \mathrm{mg} / \mathrm{dL}(\mathrm{P}=0.002)$ over time. No change in leptin or adiponectin levels was induced by any therapy. The data suggest that a low dose of simvastatin does not affect insulin resistance in overweight pre-diabetic subjects and has no effect on leptin or adiponectin levels. Further studies including a larger sample size, higher doses of statins, and a placebo control group are necessary to confirm the present data.
\end{abstract}

Key words - Simvastatin

- Metformin

- Insulin resistance

- Leptin

- Adiponectin 


\section{Introduction}

The benefits of lipid-lowering therapy for primary and secondary prevention of cardiovascular events have been extensively demonstrated, particularly concerning the use of statins (1-3). Their beneficial effects have been mainly attributed to the reduction of circulating LDL-cholesterol levels and oxidized LDL, and to the increase of collagen content in the plaque matrix (4). These actions retard the development of the atheroma and stabilize the plaque, minimizing the atherogenic process and thromboembolic events (4). Several studies have shown the efficacy of statins in reducing cardiovascular mortality $(3,5)$. Clinical trials in which statins were used as secondary prevention found the cardiovascular protective effect to be even higher in the subset of type 2 diabetic subjects (6). As far as we know, a study on the metabolic benefits of statins including only pre-diabetic subjects has not been conducted.

Abnormalities in glucose metabolism such as insulin resistance and/or hyperinsulinemia have been considered to be independent cardiovascular risk factors (7). It is possible that part of the cardiovascular benefits of statins for diabetic subjects may be due to their influence on insulin sensitivity in addition to their classical lipid-lowering effect. This hypothesis has been previously investigated and controversial results were obtained $(8,9)$. Using several techniques to measure insulin resistance, some studies have suggested that simvastatin might improve insulin sensitivity (8-10). Among the pleiotropic effects of statins, anti-inflammatory properties have been the most investigated $(11,12)$ and their cardiovascular protection has also been attributed to such actions (12, 13). A reduction in the levels of adipocytokines such as TNF- $\alpha$ induced by statin therapy (14) could be a possible mechanism improving glucose metabolism in insulin-resistant subjects. However, the impact of statins on hormones implicated in insulin sensitivity has been poorly investigated.

The relationship between insulin and leptin (an adipose tissue-derived hormone) levels is well known, the latter being involved in the pathophysiology of insulin resistance (15). Insulin-resistant obese subjects commonly exhibited hyperleptinemia that has been interpreted as a state of leptin resistance (16). On the other hand, levels of a more recently identified hormone - adiponectin (17), also secreted by adipocytes, are reduced in obesity and type 2 diabetes (16). Additionally, adiponectin is associated with low triglyceride and high HDL-cholesterol levels independently of body weight (18). A negative association of this hormone with $\mathrm{C}$ reactive protein (CRP) and fibrinogen has been demonstrated (19). These anti-inflammatory effects of adiponectin are also typical of statin therapy, raising the hypothesis that such agents could act, at least in part, via adiponectin.

The effects of simvastatin on hormones involved in glucose metabolism (insulin, leptin and adiponectin) in pre-diabetic subjects have not been investigated. Conversely, the cardioprotective benefits of metformin (20), as well as its potential to improve insulin sensitivity and to prevent overt type 2 diabetes (21), have been well documented in epidemiological studies.

The aim of the present study was to investigate the effects of a 16-week period of simvastatin treatment on insulin sensitivity and on leptin, adiponectin, and CRP levels, as compared with metformin, in two subsets of overweight pre-diabetic subjects. We hypothesized that, after simvastatin therapy, these insulin-resistant subjects could obtain hormonal benefits similar to those expected with metformin.

\section{Subjects and Methods}

Patients aged 18 to 70 years with a body mass index (BMI) of 25 to $40 \mathrm{~kg} / \mathrm{m}^{2}$ were 
recruited from the Metabolic Syndrome Outpatient Clinic of the Federal University of São Paulo. In order to include insulin-resistant subjects, those with a diagnosis of impaired fasting glucose or impaired glucose tolerance during an oral glucose tolerance test were selected (22). Written informed consent was obtained from all participants and the study was approved by the Institutional Ethics Committee. Exclusion criteria were severe dyslipidemia (LDL-cholesterol $\geq 160 \mathrm{mg} / \mathrm{dL}$ and/or triglycerides $\geq 300 \mathrm{mg} /$ $\mathrm{dL}$ ), unstable blood pressure (>140 x 90 $\mathrm{mmHg}$ ), coronary artery disease, liver or kidney failure, secondary causes of obesity, allergy or adverse effects of simvastatin or metformin, and current use of medications known to affect inflammatory activity and/ or lipid and glucose metabolism (other than the agents under investigation). No formal sample calculation was done in advance. Statistical power was calculated a posteriori based on the results and main effects of the drugs investigated. The power of interaction effect was greater than $99.9 \%$ for LDL-cholesterol and equal to $78.6 \%$ for fasting plasma glucose. Of the 44 patients enrolled, 23 were randomized to take metformin and 21 to take simvastatin. Two patients discontinued metformin therapy due to adverse effects and one simvastatin-treated patient was lost to follow-up. The baseline characteristics of the dropout subjects and those who completed the study were not significantly different (data not shown).

\section{Study protocol}

This interventional open-label study with two parallel treatment groups - metformin or simvastatin - had a duration of 18 weeks, including a 2-week run-in period when patients received dietary counseling and were encouraged to practice physical activity. Screening laboratory tests included plasma glucose (fasting and $2 \mathrm{~h}$ after a 75 -g glucose load), lipoproteins, aspartate aminotrans- ferase, alanine aminotransferase, bilirubins, electrolytes, thyroid-stimulating hormone, and electrocardiogram. At randomization, blood samples were obtained for plasma glucose, lipid profile, CRP, insulin, leptin, and adiponectin determinations. Patients were then randomized (simple randomization) to receive metformin $\left(\right.$ Glifage $\left.^{\circledR}\right) 850$ mg, twice a day, or simvastatin $\left(\right.$ Clinfar $\left.^{\circledR}\right) 20$ $\mathrm{mg}$, once a day; medications were provided by Merck S.A. (Rio de Janeiro, RJ, Brazil). The laboratory tests were repeated after 16 weeks of each therapy.

BMI was calculated as weight $(\mathrm{kg}) \mathrm{di}-$ vided by height (m) squared; waist circumference was obtained at the umbilicus level. Blood pressure was measured with a standard sphygmomanometer after $5 \mathrm{~min}$ of rest in the sitting position. Hypertension was defined as systolic and/or diastolic blood pressure levels $\geq 140 \times 90 \mathrm{mmHg}$ or the use of antihypertensive medication.

Plasma glucose, bilirubins, enzymes, and electrolytes were determined by routine methods. Cholesterol contents of lipoprotein fractions and triglycerides were measured enzymatically and processed with an automatic analyzer. High sensitivity CRP was determined by a chemiluminescent immunometric assay (Immulite, DPC, Los Angeles, CA, USA), with sensitivity of 0.01 $\mathrm{mg} / \mathrm{dL}$, intra-assay coefficient of variation of $4.2-6.4 \%$, and inter-assay coefficient of variation of $4.8-10.0 \%$. Patients with CRP greater than $1 \mathrm{mg} / \mathrm{dL}$ were excluded from the analysis of inflammatory parameters since these levels denote clinical inflammation (23). Sera were stored at $-20^{\circ} \mathrm{C}$ for further hormonal determinations. Insulin was determined by a monoclonal antibody-based immunofluorimetric assay (AutoDelfia, Perkin Elmer Life Sciences Inc., Norton, OH, USA). Insulin resistance was determined by the homeostasis model assessment method according to Matthews et al. (24) and insulin sensitivity index $\left(\mathrm{ISI}_{0,120}\right)$ was determined by the method of Gutt et al. (25). Leptin and 
adiponectin were determined by radioimmunoassay (Linco Research, St. Charles, MO, USA) with sensitivities of 0.5 and 1.0 $\mathrm{ng} / \mathrm{mL}$, respectively. The intra- and interassay CV was 3.4-8.3 and 3.0-6.2\% for leptin and 1.8-6.2 and 6.9-9.2\% for adiponectin.

\section{Statistical analysis}

Data are reported as means \pm SD for numerical variables or as number and percent for categorical variables. The paired Student $t$-test was used to compare mean clinical and laboratory data between subgroups of patients according to the type of therapy. The effect of drugs over time was assessed by analysis of variance (ANOVA) for repeated measures. Correlation between variables was tested by the Pearson coefficient and $95 \%$ confidence intervals are presented. The level of significance was set at $\alpha$ $=0.05$. Data analysis was performed using the Statistical Analysis System (SAS) software, version 8.02.

Table 1. Baseline characteristics of patients treated with metformin or simvastatin for 16 weeks.

$$
\text { Metformin }(N=21) \quad \text { Simvastatin }(N=20)
$$

Men:women

Age (years)

Body mass index $\left(\mathrm{kg} / \mathrm{m}^{2}\right)$

Waist circumference $(\mathrm{cm})$

Hypertension (\%)

Mean blood pressure $(\mathrm{mmHg})$

Disturbance of glucose metabolism (\%)

Isolated IFG

Impaired glucose tolerance

Fasting plasma glucose $(\mathrm{mg} / \mathrm{dL})$

2-h plasma glucose ( $\mathrm{mg} / \mathrm{dL})$

Fasting plasma insulin $(\mu \mathrm{U} / \mathrm{mL})$

2-h plasma insulin $(\mu \mathrm{U} / \mathrm{mL})$

Total cholesterol $(\mathrm{mg} / \mathrm{dL})$

LDL-cholesterol $(\mathrm{mg} / \mathrm{dL})$

HDL-cholesterol ( $\mathrm{mg} / \mathrm{dL})$

Triglycerides (mg/dL)

Data are reported as number, percent of patients or means $\pm \mathrm{SD}$. IFG $=$ impaired fasting glycemia.

${ }^{*} \mathrm{P}<0.05$ compared to the metformin group (ANOVA for repeated measures).

\section{Results}

Forty-one patients completed the study and their main baseline characteristics are detailed in Table 1. Except for a higher mean BMI for the metformin-treated patients at baseline, the simvastatin and metformin groups were similar regarding sociodemographic data, frequency of hypertension and glucose metabolism disturbances, as well as mean values of blood pressure, fasting and 2-h plasma glucose, fasting insulin, leptin, adiponectin, and CRP. Table 2 presents a comparison between simvastatin and metformin delta values for the selected variables. As expected, when compared to simvastatin, metformin therapy resulted in significant reductions in mean BMI and fasting plasma glucose, whereas simvastatin treatment resulted in significantly reduced total and LDL-cholesterol levels. Aspartate aminotransferase and alanine aminotransferase did not change with either treatment (data not shown). Independently of medication type, a significant decrease in CRP levels was detected from baseline to the end of the study. CRP showed a mean reduction of $0.12 \pm 0.04 \mathrm{mg} / \mathrm{dL}(\mathrm{P}=0.002)$ over time.

The insulin resistance index as determined by homeostasis model assessment decreased significantly only in metformintreated patients, whereas $\operatorname{ISI}_{0,120}$ remained unchanged with both drugs (Table 2). Mean leptin and adiponectin concentrations did not change in either treatment group (Table 2).

\section{Discussion}

This study focused on poorly investigated aspects of statin and metformin therapies such as their impact on certain hormones (leptin and adiponectin), as well as on indices of insulin sensitivity. Since both medications - simvastatin and metformin may have metabolic and cardiovascular benefits beyond their classical actions on glucose and lipid metabolism, the present study 
investigated possible hormonal effects that could be useful for patients with mild metabolic abnormalities. However, no medication was associated with changes in adiponectin or leptin levels, nor did simvastatin improve insulin sensitivity in the study sample. The expected benefit of metformin for insulin resistance was detected. Our data also confirmed previous evidence $(12,13,20)$ that both medications may have pleiotropic effects, since reduction in CRP was observed.

In addition to the main mechanism by which statins reduce cardiovascular risk (lipid-lowering effect), their ability to decrease CRP could add important prognostic information to the atherogenic process (26). CRP appears to be the strongest novel cardiovascular risk factor identified so far but also hyperinsulinemia and/or insulin resistance have been shown to be independently predictive of cardiovascular disease (7). A potential link between atherosclerosis and disturbance of glucose metabolism may involve inflammatory factors. Whether any statin plays a relevant role in insulin sensitivity in insulin-resistant subjects has been poorly investigated. This hypothesis was raised based on the finding of a lower incidence of diabetes in subjects under statin therapies in epidemiological studies (27). However, the findings of the present study did not support such a hypothesis, despite the reduction in the inflammatory marker CRP.

In contrast, many lines of evidence confirm that metformin improves insulin sensitivity and prevents diabetes in high-risk subjects (21). In the present study, we selected a subset of patients who were supposed to achieve great benefits from the attenuation of insulin resistance. Overweight pre-diabetic patients are eligible for intervention in order to minimize cardiovascular risk. This was true for the subset treated with metformin in the present study, since reductions in BMI, plasma glucose and insulin resistance were observed. The lack of benefits for glucose metabolism with simvastatin thera- py could be attributed to limitations of the study design. The 20-mg dose may have been too low to demonstrate an improvement in insulin resistance; additionally, the techniques employed to measure insulin sensitivity may have been inappropriate considering the sample size. As a consequence of this negative finding, we were unable to investigate the possible involvement of hormones (leptin and adiponectin) in the benefits of statin for glucose metabolism, as proposed.

Compliance with the medications did not seem to be a limitation, since the main expected effects of the drugs (reduction in LDL-cholesterol by simvastatin and in plasma glucose by metformin) were verified. Therefore, we cannot exclude the possibility that simvastatin has truly no impact on insulin resistance. In type 2 diabetic subjects, some investigators found that simvastatin combined with ACE inhibitors or angiotensin receptor antagonists increased adiponectin levels and insulin sensitivity, but not when administered as monotherapy $(28,29)$. Our findings are in the same line of other

Table 2. Comparison of delta values (mean difference between final and baseline values) of clinical and laboratory variables in the groups treated with simvastatin and metformin.

\begin{tabular}{lcc}
\hline & Metformin & Simvastatin \\
\hline Body mass index $\left(\mathrm{kg} / \mathrm{m}^{2}\right)$ & $1.2 \pm 0.3$ & $0.3 \pm 0.3^{*}$ \\
Waist circumference $(\mathrm{cm})$ & $2.4 \pm 0.8$ & $0.2 \pm 0.8$ \\
Mean blood pressure $(\mathrm{mmHg})$ & $0.3 \pm 2.5$ & $0.6 \pm 2.7$ \\
Fasting plasma glucose $(\mathrm{mg} / \mathrm{dL})$ & $5.8 \pm 2.7$ & $-5.1 \pm 2.7^{*}$ \\
Fasting insulin $(\mu \mathrm{U} / \mathrm{mL})$ & $6.4 \pm 3.1$ & $-0.2 \pm 3.2$ \\
HOMA-IR & $1.9 \pm 0.9$ & $-0.3 \pm 0.9^{*}$ \\
ISI 0 ,120 & $-4.1 \pm 3.5$ & $-1.8 \pm 3.6$ \\
LDL-cholesterol $(\mathrm{mg} / \mathrm{dL})$ & $4.4 \pm 5.4$ & $46.4 \pm 5.5^{*}$ \\
HDL-cholesterol $(\mathrm{mg} / \mathrm{dL})$ & $-4.0 \pm 1.5$ & $-3.0 \pm 1.6$ \\
Triglycerides $(\mathrm{mg} / \mathrm{dL})$ & $14.9 \pm 12.1$ & $28.3 \pm 12.4$ \\
Total cholesterol $(\mathrm{mg} / \mathrm{dL})$ & $4.0 \pm 5.0$ & $51.5 \pm 5.1^{*}$ \\
C-reactive protein $(\mathrm{mg} / \mathrm{dL})$ & $0.2 \pm 0.1$ & $0.1 \pm 0.1$ \\
Leptin $(\mathrm{ng} / \mathrm{mL})$ & $14.5 \pm 10.8$ & $5.8 \pm 11.0$ \\
Adiponectin $(\mu \mathrm{g} / \mathrm{mL})$ & $-0.1 \pm 0.8$ & $1.1 \pm 0.9$ \\
\hline
\end{tabular}

Data are reported as mean \pm SD. HOMA-IR $=$ homeostasis model assessment of insulin resistance; $\mathrm{ISI}_{0,120}=$ insulin sensitivity index.

${ }^{*} \mathrm{P}<0.05$ compared to the metformin group (ANOVA for repeated measures). 
reports which did not show changes in leptin or adiponectin levels following isolated statin therapy in dyslipidemic or type 2 diabetic subjects (28-30).

The expected action of metformin in improving insulin sensitivity was observed in the present study. Despite a metformin-induced weight loss and probably fat mass loss, this medication did not induce changes in leptin concentrations. Contrasting findings were observed in other studies which detected a reduction in leptin levels in obese type 2 diabetic patients (31). Our inability to demonstrate an effect of metformin therapy on leptin and adiponectin levels in metabolic disturbed subjects is in agreement with other studies (32-34). In fact, mechanisms underlying the improvement in insulin resistance induced by metformin seem to depend essentially on hepatic actions but not on adipose tissue, the main source of the latter hormones.

The lack of a placebo control group could be seen as a weak point of the present study. In fact, such group could contribute to reaching a more definite interpretation of the mechanism of action of the drugs. However, it should be mentioned that the original objective of the present study was to compare drugs with distinct mechanisms of action on metabolism. The sample size could be a second limitation to adequately evaluate the impact of the drugs on hormonal parameters.

Our data neither support that low-dose simvastatin could affect insulin resistance in overweight pre-diabetic subjects, nor show any effect of the drug on leptin or adiponectin levels. Therefore, the reported cardiovascular protective effect of statins seems to be independent of benefits for glucose metabolism. Attenuation of the inflammatory process induced by statin and metformin could be an additional mechanism improving cardiovascular risk. Further studies including larger samples and higher daily doses of medications are necessary.

\section{References}

1. Brown G, Albers JJ, Fisher LD, Schaefer SM, Lin JT, Kaplan C, et al. Regression of coronary artery disease as a result of intensive lipidlowering therapy in men with high levels of apolipoprotein B. N Engl J Med 1990; 323: 1289-1298.

2. Gotto AM Jr, Grundy SM. Lowering LDL cholesterol: questions from recent meta-analyses and subset analyses of clinical trial Data Issues from the Interdisciplinary Council on Reducing the Risk for Coronary Heart Disease, Ninth Council Meeting. Circulation 1999; 99: E1-E7.

3. Anonymous. Randomised trial of cholesterol lowering in 4444 patients with coronary heart disease: the Scandinavian Simvastatin Survival Study (4S). Lancet 1994; 344: 1383-1389.

4. Crisby M, Nordin-Fredriksson G, Shah PK, Yano J, Zhu J, Nilsson J. Pravastatin treatment increases collagen content and decreases lipid content, inflammation, metalloproteinases, and cell death in human carotid plaques: implications for plaque stabilization. Circulation 2001; 103: 926-933.

5. Kinlay S, Schwartz GG, Olsson AG, Rifai N, Leslie SJ, Sasiela WJ, et al. Effects of atorvastatin on early recurrent ischemic events in acute coronary syndromes: the MIRACL study: a randomized controlled trial. JAMA 2001; 285: 1711-1718.

6. Heart Protection Study Collaborative Group. MRC/BHF Heart Protection Study of cholesterol lowering with simvastatin in 20,536 high-risk individuals: a randomised placebo-controlled trial. Lancet 2002; 360: 7-22.
7. Despres JP, Lamarche B, Mauriege P, Cantin B, Dagenais GR, Moorjani $S$, et al. Hyperinsulinemia as an independent risk factor for ischemic heart disease. N Engl J Med 1996; 334: 952-957.

8. Paolisso G, Barbagallo M, Petrella G, Ragno E, Barbieri M, Giordano $M$, et al. Effects of simvastatin and atorvastatin administration on insulin resistance and respiratory quotient in aged dyslipidemic noninsulin dependent diabetic patients. Atherosclerosis 2000; 150: 121127.

9. Ohrvall M, Lithell H, Johansson J, Vessby B. A comparison between the effects of gemfibrozil and simvastatin on insulin sensitivity in patients with non-insulin-dependent diabetes mellitus and hyperlipoproteinemia. Metabolism 1995; 44: 212-217.

10. Farrer M, Winocour PH, Evans K, Neil HA, Laker MF, Kesteven P, et al. Simvastatin in non-insulin-dependent diabetes mellitus: effect on serum lipids, lipoproteins and haemostatic measures. Diabetes Res Clin Pract 1994; 23: 111-119.

11. Ridker PM, Rifai N, Stampfer MJ, Hennekens CH. Plasma concentration of interleukin- 6 and the risk of future myocardial infarction among apparently healthy men. Circulation 2000; 101: 1767-1772.

12. Jialal I, Stein D, Balis D, Grundy SM, Adams-Huet B, Devaraj S. Effect of hydroxymethyl glutaryl coenzyme a reductase inhibitor therapy on high sensitive C-reactive protein levels. Circulation 2001; 103: 1933-1935.

13. Ridker PM, Rifai N, Pfeffer MA, Sacks FM, Moye LA, Goldman S, et al. Inflammation, pravastatin, and the risk of coronary events after 
myocardial infarction in patients with average cholesterol levels. Cholesterol and Recurrent Events (CARE) Investigators. Circulation 1998; 98: 839-844.

14. Solheim S, Seljeflot I, Arnesen H, Eritsland J, Eikvar L. Reduced levels of TNF alpha in hypercholesterolemic individuals after treatment with pravastatin for 8 weeks. Atherosclerosis 2001; 157: 411 415.

15. Caro JF, Sinha MK, Kolaczynski JW, Zhang PL, Considine RV. Leptin: the tale of an obesity gene. Diabetes 1996; 45: 1455-1462.

16. Bulcão C, Ferreira SRG, Giuffrida FMA, Ribeiro-Filho FF. The new adipose tissue and adipocytokines. Curr Diabetes Rev 2006; 2: 1928.

17. Kihara S, Arita Y, Ouchi N. A novel adipocyte-derived factor, adiponectin, inhibits growth of vascular smooth cells. Int $J$ Obes 1998; 22 (Suppl 3): O16-pS5.

18. Baratta R, Amato S, Degano C, Farina MG, Patane G, Vigneri R, et al. Adiponectin relationship with lipid metabolism is independent of body fat mass: evidence from both cross-sectional and intervention studies. J Clin Endocrinol Metab 2004; 89: 2665-2671.

19. Fernandez-Real JM, Lopez-Bermejo A, Casamitjana R, Ricart W Novel interactions of adiponectin with the endocrine system and inflammatory parameters. J Clin Endocrinol Metab 2003; 88: 27142718.

20. UK Prospective Diabetes Study (UKPDS) Group. Effect of intensive blood-glucose control with metformin on complications in overweight patients with type 2 diabetes (UKPDS 34). Lancet 1998; 352: 854865.

21. Knowler WC, Barrett-Connor E, Fowler SE, Hamman RF, Lachin JM, Walker EA, et al. Reduction in the incidence of type 2 diabetes with lifestyle intervention or metformin. N Engl J Med 2002; 346: 393-403.

22. American Diabetes Association. Diagnosis and classification of diabetes mellitus. Diabetes Care 2006; 29 (Suppl 1): S43-S48.

23. Pearson TA, Mensah GA, Alexander RW, Anderson JL, Cannon RO III, Criqui $M$, et al. Markers of inflammation and cardiovascular disease: application to clinical and public health practice: A statement for healthcare professionals from the Centers for Disease Control and Prevention and the American Heart Association. Circulation 2003; 107: 499-511.

24. Matthews DR, Hosker JP, Rudenski AS, Naylor BA, Treacher DF, Turner RC. Homeostasis model assessment: insulin resistance and beta-cell function from fasting plasma glucose and insulin concen- trations in man. Diabetologia 1985; 28: 412-419.

25. Gutt M, Davis CL, Spitzer SB, Llabre MM, Kumar M, Czarnecki EM, et al. Validation of the insulin sensitivity index (ISI $(0,120))$ : comparison with other measures. Diabetes Res Clin Pract 2000; 47: 177184.

26. Ridker PM, Rifai N, Rose L, Buring JE, Cook NR. Comparison of Creactive protein and low-density lipoprotein cholesterol levels in the prediction of first cardiovascular events. N Engl J Med 2002; 347: 1557-1565.

27. Freeman DJ, Norrie J, Sattar N, Neely RD, Cobbe SM, Ford I, et al. Pravastatin and the development of diabetes mellitus: evidence for a protective treatment effect in the West of Scotland Coronary Prevention Study. Circulation 2001; 103: 357-362.

28. Koh KK, Quon MJ, Han SH, Ahn JY, Jin DK, Kim HS, et al. Vascular and metabolic effects of combined therapy with ramipril and simvastatin in patients with type 2 diabetes. Hypertension 2005; 45: 1088-1093.

29. Koh KK, Quon MJ, Han SH, Chung WJ, Ahn JY, Seo YH, et al. Additive beneficial effects of losartan combined with simvastatin in the treatment of hypercholesterolemic, hypertensive patients. Circulation 2004; 110: 3687-3692.

30. Bayes B, Granada ML, Lauzurica R, Pastor MC, Navarro M, Bonet $\mathrm{J}$, et al. Effect of low doses of atorvastatin on adiponectin, glucose homeostasis, and clinical inflammatory markers in kidney transplant recipients. Transplant Proc 2005; 37: 3808-3812.

31. Komori T, Yoshida F, Nakamura J, Miyazaki S, Miura H, Iguchi A. Metformin ameliorates treatment of obese type 2 diabetic patients with mental retardation; its effects on eating behavior and serum leptin levels. Exp Clin Endocrinol Diabetes 2004; 112: 422-428.

32. Ciaraldi TP, Kong AP, Chu NV, Kim DD, Baxi S, Loviscach M, et al. Regulation of glucose transport and insulin signaling by troglitazone or metformin in adipose tissue of type 2 diabetic subjects. Diabetes 2002; 51: 30-36.

33. Fujita $\mathrm{H}$, Fujishima $\mathrm{H}$, Koshimura J, Hosoba M, Yoshioka N, Shimotomai $\mathrm{T}$, et al. Effects of antidiabetic treatment with metformin and insulin on serum and adipose tissue adiponectin levels in $\mathrm{db} / \mathrm{db}$ mice. Endocr J 2005; 52: 427-433.

34. Tiikkainen M, Hakkinen AM, Korsheninnikova E, Nyman T, Makimattila S, Yki-Jarvinen $\mathrm{H}$. Effects of rosiglitazone and metformin on liver fat content, hepatic insulin resistance, insulin clearance, and gene expression in adipose tissue in patients with type 2 diabetes. Diabetes 2004; 53: 2169-2176. 